\title{
OSTI
}

\section{Emittance Growth from Transient Coherent Synchrotron Radiation}

\author{
C. L. Bohn, R. Li, and J. J. Bisognano \\ Thomas Jefferson National Accelerator Facility \\ 12000 Jefferson Avenue, Newport News, VA 23606 (USA)
}

\begin{abstract}
If the energies of the individual particles comprising a bunch change as the bunch traverses a bending system, even if it is achromatic, then betatron oscillations can be excited. Consequently, the transverse emittance of the bunch. will grow as it moves downstrenm. Short bunches may be particularly susceptible to emission of coherent synchrotron radiation which can act back on the particles to change their energies and trajectories. Because a bend spans a well-defined length and angle, the bunch-excited wakefield and its effect back on the bunch are inherently transient. We outline a recently developed theory of this effect and apply it to example bending systems.
\end{abstract}

\section{Introduction}

When accelerated, a compact ensemble, or bunch, of relativistic charged particles radiates coherently at wavelengths comparable to or longer than the bunch length. This establishes an electromagnetic wake that acts back on the bunch and distorts it. For example, coherent synchrotron radiation (CSR) establishes an electromagnetic-field gradient along the bunch and causes the energies of the individual particles comprising the bunch to change by differing amounts as the bunch traverses a magnetic bend. In tum, even in a magnetic bending system that is achromatic, the transverse emittance of the bunch grows as it moves downstream.

CSR-induced beam degradation is of concern for any accelerator of short, high-charge bunches that incorporates magnetic bends in its transport system. This includes sccelerator drivers for high-power freo-electron lasers. For example, in the design of Jefferson Lab's high-power infrared FEL, or "TR Demo" [1], which incorporates a tronsport system to recinculate the electron beam back to the linac for energy recovery, potential beam degradation due to CSR motivated placing the wiggler directly after the linac rather than after the first recirculation bend, with a concomitant increase in the machine footprint. This exemplifies the impact of CSR on accelerator desigen and highlights the need for detailed understanding of the phenomenon.

\section{Fighlights of a Theory}

In a recent paper [2], hereafter called Paper 1, we formulated an analytic theory of CSR. The theory includes transient effects and an infinite parallel-plate model of the vacuum pipe, and it uses a rigid line charge as a model of the bunch The calculation of the normalized transverse emittance includes a proper treatment of the transport lattice. Details of the calculations, and resulting analytic expressions for the radiated power and the normalized transverse emittance, appear in Paper 1.

A representative result of a calculation of the power radiated in the presence of the parallel plates is shown in Figure 1. The selected parameters correspond to a large vacuum chamber (having the characteristic dimension of the vacuum chrmber planned for the IR Demo) and ahort bunch (also representative of that in the IR Demo). The

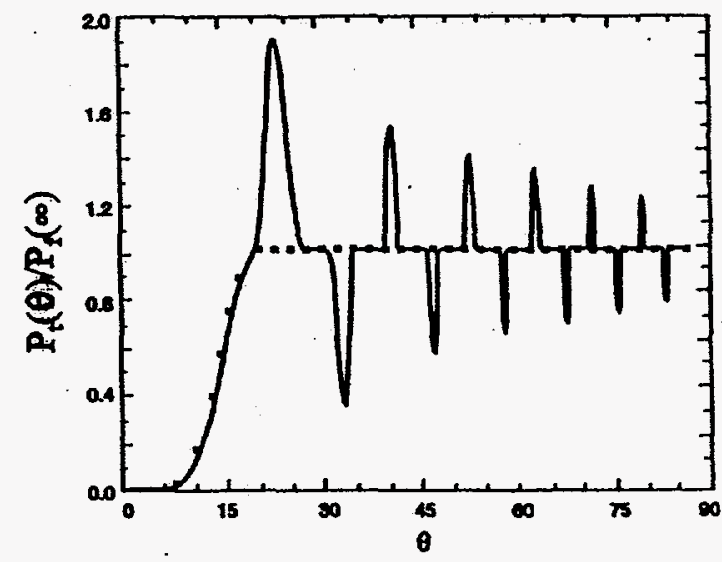

Fig 1. The dotted curve denotes the transieat freospace power $P_{1}(\theta) P_{\{}(\infty)$ radiated by a bunch in a bend va bend angle $\theta$. The solid curve denotes the transient radisted powrer $R_{2}(\theta) / P_{1}(\infty)$ in parallel onducting plates. Parameters are: $1 \mathrm{~m}$ bend radius, 40 $\mathrm{MeV}$ beam energy, $5 \mathrm{~cm}$ plate spacing and $0.5 \mathrm{~mm}$ bunch lengh. 


\section{:ECEIVED \\ OCT 031996 \\ OSTI}

This report has been reproduced from the best available copy.

Available to DOE and DOE contractors from the Office of Scientific and Technical Information, P.O. Box 62, Oak Ridge, TN 37831; prices available from (615)576-8401, FTS 626-8401.

Available to the public from the National Technical Information Service, U.S. Department of Commerce, 5285 Port Royal Rd., Springfield, VA 22161.

Price: Printed Copy AO2

Microfiche AO1 


\section{DISCLAIMER}

This report was prepared as an account of work sponsored by an agency of the United States Government. Neither the United States Government nor any agency thereof, nor any of their employees, makes any warranty, express or implied, or assumes any legal liability or responsibility for the accuracy, completeness, or usefulness of any information, apparatus, product, or process disclosed, or represents that its use would not infringe privately owned rights. Reference herein to any specific commercial product, process, or service by trade name, trademark, manufacturer, or otherwise does not necessarily constitute or imply its endorsement, recommendation, or favoring by the United States Government or any agency thereof. The views and opinions of authors expressed herein do not necessarily state or reflect those of the United States Government or any agency thereof. 
DISCLAIMER

Portions of this document may be illegible in electronic image products. Images are produced from the best available original document. 
localized transient peaks are due to the interaction of the bunch with its image charges generated by the parallel plates. A small vacuum chamber and long bunch gives rise to considerable shielding of the steady-state radiation (bend angle $\theta \rightarrow \infty$ ), but this is not the situation illustrated in Figure 1, and so shielding is negligible there.

Inspection of Figure 1 reveals general qualitative aspects of the effect of CSR on transverse-emittance growth in the presence of conducting walls. First, in short bends, the existence of a formation length to reach steady state will cause the emittance growth to be less than that predicted from steady-state CSR. Second, in long bends, the transients will tend to average out and the steady-state radiation field will predominate.

\section{Applications of the Theory}

Paper 1 gives a recipe for calculating the transversoemittance growth resulting from the CSR-induced electromagnetic field across the rigid-line bunch. The transverse emittance is $\varepsilon=\left[\varepsilon_{0}^{2}+(\Delta \varepsilon)^{2}\right]^{1 / 2}$, with $\varepsilon_{0}$ the initial emittance and $\Delta z$ the CSR-induced emittance growth. If the dominant effect is to change the energies of the individual particles as the bunch traverses a bend magnet, then $\left.\Delta \varepsilon \approx \gamma\left[\left\langle x^{2}\right\rangle \delta x^{\prime 2}\right\rangle\right]^{1 / 2}$, in which $\gamma$ is the design energy, $x$ is the transverse offset from the design orbit, and $\delta x^{\prime}=\Delta x^{\prime}-\left\langle\Delta x^{\prime}\right\rangle$, where $\Delta x^{\prime}$ is the CSR-induced dispersive angle. The CSR-induced emittance growth scales proportionally to $\gamma^{-1} I_{f}(R / \sigma)^{1 / 3}$, where $I_{p}$ is the peak current, $R$ is the bend radius, and $\sigma$ is the rms bunch length. It also sciles proportionally to a time-dependent coefficient accounting for the transient behavior, and a bend-angle-dependent coefficient which scales as $\theta^{2}$ for short bends and as $\theta \sin \theta+\cos \theta-1 \approx \theta \sin \theta$ for long bends.

Wo have applied the results of Paper 1 to calculate the emittance growth in bending subsystems of the IR Demo. The buncher chicane preceding the wiggier is comprised of four short magnets, as shown in Figure 2. A tracking code was used to calculate the bunch length at each magnet. The transport matrices of the lattice were used to propagate an electron through the chicine while using the CSR theory to calculate the electron position and energy offsets at the exit of each magent assuming they are uncorrelated with respect to CSR. The finil normalized emittance was then calculated using the results for the electron's final offset and angle. It was calculated to be about $7 \%$ higher thm the nominal initial normalized emittance of $6.5 \mathrm{~mm}$-mrad. By comparison, if a steady-state force had been assumed, the final emittance works out to be about $43 \%$ higher than the nominal initial emittance. This illustrates the beneficial consequence of the formation length in short bend magnets.

We also applied this procedure to the $180^{\circ}$ bend magnet of the IR Demo's first recirculation bend. In this case the steady-state force suffices for the calculation. According to particle-tracking results, the bunch length varies dramatically during transit. A rough estimate using a $1 \mathrm{~mm}$ representative average bunch length yields a dangerously large factor-af-two emittance growth.
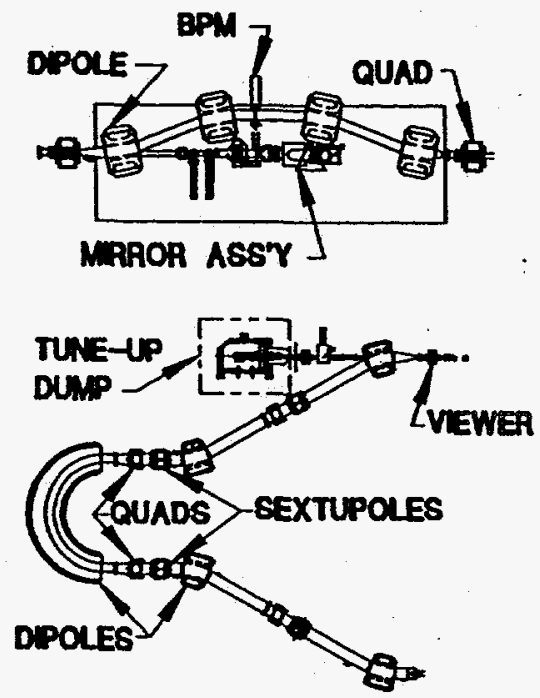

Fig 2. Buncher chicane precoding the wiggier in Jefferson Lob's IR Demo (top), and the first recircailetion bend (bottom).

\section{Future Work}

Although Paper 1 incorporates several aspects of CSR physics missing in preceding work, it also uses many simplifications. Chief among these is inherent in the "rigid-line-charge" model and the omission of the local coulomb force. It still remains to incorporate selfconsistency and the threo-dimensional propecty of the bunch. It also remains to include all of the effects of both the global CSR and local coulomb forces on the bunch dynamics, as well as to assess possible correlations between the bending magnets. We are currently exploring these intricacies in in effort to generalize the theory. We are also allowing for instrumentation in the IRFEL recirculation bend for emittance measurements to support beam-bending experiments.

This work was supported by the U. S. Department of Energy under contract DE-ACO5-84ER40150.

\section{References}

[1] S. Benson, these Proceodings

[2] R. Li, C. L Bom, and J. J. Bisognano, Phys Rev. Lett (submitted). 